\title{
Mass-disorder effects on the frequency filtering in one-dimensional discrete particle systems
}

\author{
Brian P. Lawney and Stefan Luding \\ Multiscale Mechanics (MSM), MESA+, Faculty of Engineering Technology \\ PO Box 217, 7500 AE Enschede, Netherlands
}

\begin{abstract}
.
We numerically study the effects of mass-disorder on the signal transmission properties of one-dimensional linearly coupled granular systems. By driving such systems at a specified input frequency, we are able to investigate the disorderinduced high-frequency filtering of signals downstream from the excitation source. We consider ensembles of systems composed of random masses selected from normal, uniform, and binary distributions and find that the transmitted frequency content is not sensitive to the particular distribution or arrangement of the random masses. Rather, only knowledge of the moments of the mass distribution is necessary to quantify the bandwidth of transmitted frequencies.
\end{abstract}

Keywords: Signal transmission, one-dimensional chains, frequency filtering, random

PACS: $81.05 . \mathrm{Rm}, 43.20 .+\mathrm{g}, 46.40 . \mathrm{Cd}$

\section{INTRODUCTION}

Simplified, one-dimensional models are often exploited for their potential to expose the relevant physics of more general, higher dimensional systems [1]. Indeed, one-dimensional models of discrete systems have received significant attention in the literature [2, 3, 4, 5] with a particular emphasis on nonlinear phenomena such as soliton-like wave generation $[6,7,8,9,10,11,12]$. Additional interest has been placed on the artificial design of granular systems with the aim of shock protection or damage mitigation; arrangements employing techniques such as "decoration" [13, 14, 15], tapering $[16,17,18,19]$, combinations thereof $[13,20]$, and variation of particle material $[21,22]$ have been employed. Sen et al. [23] provide a review covering many of the studies in this area.

Only limited attention has been placed on the study of frequency-filtering effects of systems featuring random mass distributions. Mouraille and Luding [24] numerically studied high-frequency filtering of perturbed minimally polydisperse (almost) crystalline, contactdisordered geometries. Others have experimentally [25] and numerically [26] observed signatures of this behavior but the connection between the particle-scale dynamics and the system-scale behavior remains a subject warranting further study.

In this paper we develop a simple, general model for the motion of a driven, mass-disordered, pre-stressed, noncohesive linear granular chains. Following this we perform a numerical parameter study to investigate how the mass distribution affects the high-frequency filtering properties of these systems. Employing random chains composed of particles sampled from normal, uniform, and binary distributions we simulate the driven motion of these systems and analyze the motion of particles downstream from the excitation source.

\section{MODELING}

We consider one-dimensional arrays of random mass particles which interact with only their immediate neighbors in a purely repulsive manner. In addition, we consider chains that are pre-compressed such that there is some initial strain associated with the equilibrium configuration. Thus, we are not near the "sonic vacuum" limit of Nesterenko [6, 27]. The general nonlinear interaction force $\tilde{F}_{(i, j)}$ between neighboring particles $i$ and $j$ overlapping by distance $\delta$ is modeled as,

$$
\left|F_{(i, j)}\right| \propto \delta_{(i, j)}^{1+\beta}, \quad \delta_{(i, j)} \geq 0
$$

where the proportionality would be related to a "stiffness" that changes with the value of $\beta$ and depends, in general, on the properties of the contacting bodies. Assuming sufficient confining force as to approach linear coupling between particles [28], we take $\beta=0$. Linearizing about the equilibrium positions and scaling by the mean mass, stiffness, and a characteristic length scale we obtain the following equation of motion for general particle $i$ :

$$
\begin{aligned}
b^{(i)} \frac{d^{2} u^{(i)}}{d \tau^{2}} & =\kappa_{(i-1, i)}\left[\Delta_{(i-1, i)}-\left(u^{(i)}-u^{(i-1)}\right)\right] \\
& -\kappa_{(i+1, i)}\left[\Delta_{(i+1, i)}-\left(u^{(i+1)}-u^{(i)}\right)\right] .
\end{aligned}
$$


Here we define $b^{(i)} \equiv m^{(i)} / m_{o}\left(\right.$ mean mass $\left.m_{o}\right)$ and $u^{(i)}$ to be the scaled mass and displacement of the $i^{\text {th }}$ particle, and $\kappa_{(i, j)}$ and $\Delta_{(i, j)}$ as the scaled interparticle stiffness and equilibrium overlap between particles $i$ and $j$, respectively. The time $\tau$ is scaled accordingly by a characteristic time related to the natural frequency of oscillation between two particles with mean mass and stiffness. For more details and a general expression with $\beta \geq 0$ see [29].

For a chain of $N$ masses constrained between endparticles subject to the specified driven and fixed boundary conditions, we may express the $N$ linear equations in matrix form

$$
\mathbf{M} \frac{\mathrm{d}^{2} \mathbf{u}}{\mathrm{d} \tau^{2}}=\mathbf{K u}+\mathbf{f}
$$

where $\mathbf{M}$ is a diagonal matrix with the random mass ratios $b^{(1)}$ through $b^{(N)}$ on the diagonal, and $\mathbf{K}$ is a symmetric, tri-diagonal matrix. Since we look to isolate the effect of mass disorder, we assume uniform contact stiffnesses $\left(\kappa_{(i, j)}=1\right)$ and $\mathbf{K}$ simplifies to have entries of -2 on the diagonal and entries of +1 on the sub and superdiagonal. The forcing vector $\mathbf{f}$ has only a single non-zero entry of $f_{1}(\tau)=\varepsilon \sin \omega_{o} \tau$ in the first position where $\varepsilon$ and $\omega_{o}$ are the scaled displacement and frequency of the driven end particle.

Defining $\mathbf{A} \equiv-\mathbf{M}^{-1} \mathbf{K}$ and assuming normal mode motion, we solve the eigenvalue problem $\mathbf{A u}=\omega^{2} \mathbf{u}$ to obtain $N$ orthogonal eigenvectors. Assembling the $(N \times N)$ matrix $\mathbf{S}$ such that column $j$ is proportional to eigenvector $\mathbf{S}_{(j)}$, we solve (3) by transformation into the eigenvector basis. Note that $\mathbf{S}$ is additionally defined to satisfy the orthogonality relation $\mathbf{S}^{T} \mathbf{M S}=\mathbf{I}$ where $\mathbf{I}$ is the identity matrix. Exploiting this orthogonality relation and assuming $\mathbf{u}=\mathbf{0}$ and $d \mathbf{u} / d \tau=\mathbf{0}$, we obtain the displacement history of particle $p$ as

$u^{(p)}(\tau)=\varepsilon \sum_{j=1}^{N} \frac{S_{p j} S_{1 j}}{\left(\omega_{(j)}^{2}-\omega_{o}^{2}\right)}\left(\sin \omega_{o} \tau-\frac{\omega_{o}}{\omega_{(j)}} \sin \omega_{(j)} \tau\right)$,

where $\omega_{(j)}$ is the eigenfrequency (normalized by the characteristic time scale) corresponding to normal mode $\mathbf{s}_{(j)}$. Note that in the case of the perfect chain the eigenvectors are sinusoids subject to fixed boundary conditions.

For the undriven perfect (monodisperse) chain, we are able to find the dispersion relation $\omega(k)=2 \sin k$ relating the frequency and dimensionless wavenumber $k$ (scaled by particle radius). This is important because we note that perfect linear chains are capable of transmitting frequencies in the (normalized) passband $0<\omega \leq 2$.

Creation of mass-disordered chains

In this study we consider chains composed of particles selected from normal $f^{(n)}(b)$, uniform $f^{(u)}(b)$, and binary discrete $f^{(d)}(b)$ distributions.
For the normal distribution, we divide by the mean mass so that $\bar{b}=1$; the standard deviation $\xi$ is similarly scaled. We use the variable $\xi$ to quantify the disorder of the random chains. Note that the physical restriction of positive mass values results in some cutoff of the distribution. When comparing the various distributions we restrict $\xi \leq 0.5$ so that the cutoff is at most within two standard deviations of the mean. Thus, only a small portion of the distribution is missing $(\approx 2.3 \%)$. Large masses have no such cutoff and the ensemble-averaged largest mass increases linearly with $\xi$. Values of $\xi>0.5$ are simulated for normally distributed masses to investigate the effect of greater disorder, but these results are not considered in the comparison between mass distributions.

For comparison between the three distributions, we match the first two moments of the three probability density functions. By definition, the $n^{\text {th }}$ moment of a given distribution $f^{(q)}(b)$ is defined as,

$$
M_{n}^{(q)}=\int_{-\infty}^{\infty} b^{n} f^{(q)}(b) \mathrm{d} b
$$

where $q$ is used to identify the specific distribution type. Matching the moments of the normal distribution with mean $\bar{b}=1$ and standard deviation $\xi$ such that $M_{1}^{(n)}=1$ and $M_{2}^{(n)}=1+\xi^{2}$, we find the limits of the uniform distribution to be $[1-\sqrt{3} \xi, 1+\sqrt{3} \xi]$. For the binary distribution we place masses at $1 \pm \xi$. To approximately preserve symmetry about the mean, the masses are selected with equal probability; long (length $\ell$ ) monodisperse sections which might artificially affect the transmission properties of the chain have probability $\approx 2^{-\ell}$.

\section{NUMERICAL SIMULATIONS}

Following the creation of the chains of $(N+2)$ random masses, we numerically solve Eq. (3) with $\mathbf{f}=\mathbf{0}$ yielding the eigenvector matrix $\mathbf{S}$ and $N$ eigenfrequencies $\left\{\omega_{(j)}\right\}$ $(j=1, \ldots, N)$. From Eq. (4) we calculate the displacement history for particles $p$ in the range $[1, c]$ and for $\tau \leq \tau^{*}$. We choose $c$ such that no energy from the transmitted signal has reached the fixed end at $p=N$ and select the time window so that the slowest wave components have had sufficient time to reach particle $c$. The discrete time step is small enough to resolve all possible frequencies of the system.

To visualize the frequency filtering we perform a discrete Fourier transform (DFT) on each particle p's motion to obtain $U^{(p)}(\omega)$ and plot the absolute value of these normalized Fourier components in greyscale. Darker shades correspond to greater absolute values with black equivalent to a component of $\geq 0.2$. Figure 1 depicts a single chain realization for normally distributed masses excited by input frequencies (a) $\omega_{o}=3.0$ and (b) 



FIGURE 1. Propagation spectrum for a single, normally distributed disordered chain $\left(\xi=0.5, \mathrm{~d} \tau=0.0667, \tau^{*}=546.41\right.$, $N=500, c=200$ ) driven at (a) $\omega_{o}=3.0$ and (b) $\omega_{o}=1.0$. In (a), we note that the wavelengths of the "stitching patterns" for increasing $p$ and fixed $\omega$ are accurately predicted by the dispersion relation of a perfectly ordered chain.
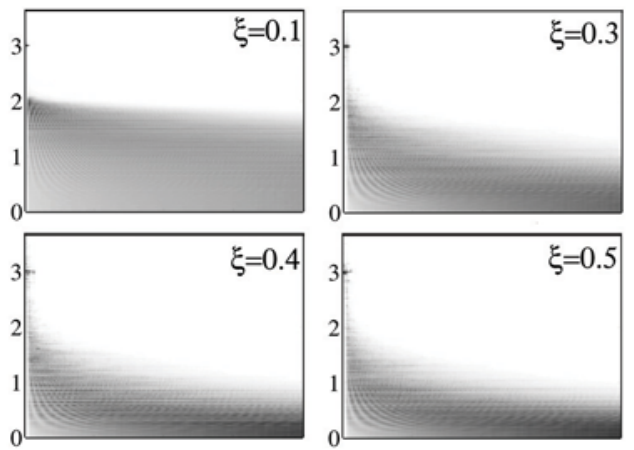

FIGURE 2. Ensemble-averaged transmission profiles for $\xi=0.1,0.3,0.4,0.5$ with $\omega_{o}=3.0$, normal distribution, and $c=200$

$\omega_{o}=1.0$. For each instance in the ensemble of 200 chains considered, we determine the frequency content at each position and subsequently average to obtain $\left\langle\left|U^{(p)}\right|\right\rangle$.

\section{DISCUSSION}

As evidenced by varying the width parameter $\xi$ in Figure 2 , increasing the magnitude of disorder in driven granular chains serves to reduce the bandwidth of the frequencies transmitted downstream of the source. In-


FIGURE 3. Comparison of mass distributions for $\xi=0.5$, $\omega_{o}=3.0$, and $c=200$

deed, the perfect (ordered) monodisperse chain permits unfiltered transmission of all frequencies in the passband $0 \leq \omega \leq 2$. Driver excitation by frequencies $\omega_{o}>2$ manifests similar to a delta-pulse excitation where all frequencies in the passband are excited equally; however, there is no penetration of frequencies outside this band. In disordered systems we observe similar rapid attenuation of the input signal, although frequencies $\omega>2$ do penetrate the system due to the presence of smaller masses which can sustain higher frequency oscillations.

The similarity of the distributions in Figure 3 is striking considering the qualitative difference in the mass distributions. Such quantitatively similar filtering is observed for a number of different disorder magnitudes less than 0.5. Further confirmation of this is provided by plotting vertical cross-sections of the plots in Figure 3 (not shown). These observations suggest that the intermediate-sized masses do not play a significant role in the filtering of high-frequency content. Rather, the interaction between the largest and smallest masses (as quantified by the moment of the distributions) is the determining factor.

Additionally, the magnitude of the largest masses alone does not seem to affect the filtering behavior in any significant manner. In simulations of normally distributed masses with $\xi$ approaching unity (data not shown), the ensemble-averaged largest mass was 1.61 times greater than for $\xi=0.5$, yet the profiles of transmitted frequen- 
cies were not appreciably different. As the disorder increased, the cutoff imposed by the $b>0$ restriction simply yielded a greater quantity of relatively smaller masses, but the absolute minimum mass did not decrease.

As examined in [29], spatial localization of the eigenmodes (Anderson localization [30]) in the presence of disorder is responsible for the filtering of high-frequency signals. Low-frequency signals are not as sensitive to the arrangement of the masses and hence eigenmodes for low-frequency disordered systems are quite similar to those of monodisperse chains (sinusoids). Evidence of this is provided in Figure 1(b) where the signal with relatively low-frequency input propagates quite far with negligible filtering to even lower frequencies.

\section{CONCLUSION}

We have presented observations from numerical simulations on driven, compressed, linear, one-dimensional granular systems. We employ such systems to provide simple insight into the behavior of higher dimensional, more general granular systems. Similar linear analyses could be performed on higher dimensional systems, but the additional spatial dimensions introduce geometrydependent effects (e.g. potentially transient contacts) that make interpretation of the results more difficult. Indeed, small size perturbations introduced in three-dimensional granular crystals $(\xi \approx 0.007)$ produces qualitatively similar frequency filtering to linear chains at far greater disorder magnitudes [24, 29] so one-dimensional models are unable to capture such complex behavior in general.

We began with a general theory of one-dimensional systems featuring nonlinear interactions (for full details see [29]) and subsequently introduced linear assumptions consistent with sufficiently compressed systems. By controlling the amount of mass-disorder in the chains we were able to qualitatively observe the high-frequency filtering properties of these systems. A significant result of our investigation is the evidence that the particular nature of disorder is relatively minor; systems composed of masses selected from normal, uniform, and binary distributions filter signals in a very similar way. Our results suggest that only specification of the first two moments of the mass-distribution is necessary to quantify the bulk transmission properties of one-dimensional driven chains. Looking to similar studies on one-dimensional oscillating systems where chains were artificially constructed to produce desired outputs (e.g. through decoration, tapering), this could also have implications for practical design and simplified modeling of more general mass-disordered systems. Future studies should investigate filtering in regimes where nonlinear effects (e.g. Hertzian contacts) are more dominant and where real three-dimensional contact networks are present.
Acknowledgment: This research is supported by VICI Project No. 10828/NWO-STW.

\section{REFERENCES}

1. E. Lieb, and D. Mattis, Mathematical Physics in One Dimension. Exactly soluble models of interacting particles., Academic Press, New York, 1966.

2. F. Dyson, Phys. Rev. 92, 1331-1338 (1953).

3. H. Schmidt, Phys. Rev. 105, 425-441 (1957).

4. P. Dean, Proc. Phys. Soc. 73, 413-421 (1959).

5. E. Montroll, and R. Potts, Phys. Rev. 100, 525-543 (1955)

6. V. Nesterenko, J. Appl. Mech. Tech. Phys. 24, 733-743 (1983).

7. M. Manciu, S. Sen, and A. Hurd, Physica D 157, 226-240 (2001).

8. M. Manciu, S. Sen, and A. J. Hurd, Physica A 274, 588-606 (1999).

9. M. Manciu, S. Sen, and A. J. Hurd, Physica A 274, 607-618 (1999)

10. S. Sen, M. Manciu, and J. Wright, Physical Review E 57, 2386-2397 (1998).

11. C. Coste, and B. Gilles, Eur. Phys. J. B 7, 155-168 (1999)

12. S. Job, F. Melo, A. Sokolow, and S. Sen, Gran. Matt. 10, 13-20 (2007).

13. U. Harbola, A. Rosas, A. Romero, M. Esposito, and K. Lindenberg, Phys. Rev. E 80, 051302 (2009).

14. U. Harbola, A. Rosas, A. Romero, and K. Lindenberg, Phys. Rev. E 82, 011306 (2010).

15. M. Gharib, A. Celik, and Y. Hurmuzlu, J. Appl. Mech. 78, 031005 (2011).

16. R. Doney, and S. Sen, Phys. Rev. E 72, 041304 (2005).

17. F. Melo, S. Job, F. Santibanez, and F. Tapia, Phys. Rev. E 73, 041305 (2006)

18. A. Sokolow, J. Pfannes, R. Doney, M. Nakagawa, J. Agui, and S. Sen, Appl. Phys. Lett. 87, 254104 (2005).

19. D. Wu, Physica A 315, 194-202 (2002).

20. R. Doney, and S. Sen, Physical Review Letters 97, APS - 4 (2006).

21. C. Daraio, V. Nesterenko, E. Herbold, and S. Jin, Phys. Rev. Lett. 96, 058002 (2006).

22. E. Herbold, J. Kim, V. Nesterenko, S. Wang, and C. Daraio, Acta Mech. 205, 85-103 (2009).

23. S. Sen, J. Hong, J. Bang, E. Avalos, and R. Doney, Physics Reports 462, 21-66 (2008).

24. O. Mouraille, and S. Luding, Ultrasonics 48, 498-505 (2008).

25. X. Jia, C. Caroli, and B. Velicky, Physical Review Letters 82, 1863-1866 (1999).

26. J. Judge, B. Houston, D. Photiadis, and P. Herdic, Journal of Sound and Vibration 290, 1119-1140 (2006).

27. L. Gomez, A. Turner, M. van Hecke, and V. Vitelli, Phys. Rev. Lett. 108, 058001 (2012)

28. R. S. Sinkovits, and S. Sen, Phys. Rev. Lett. 74, 2686 (1995).

29. B. Lawney, and S. Luding, to be published (2013).

30. P. Anderson, Phys. Rev. 109, 1492-1505 (1958). 